\title{
MS11-03 | EXI, THE EXTENDED ISPyB INTERFACE
}

Santoni, Gianluca (ESRF, Grenoble, FRA)

The ISPyB database and web interface have been the mainstays of a Laboratory Information Management System for structural biology experiments which has been in use at the ESRF since 2001. EXI is an upgraded interface for ISPyB, currently used in the context of MX, bioSAXS and Cryo EM experiments and which allows the preparation of synchrotron experiments from the home laboratory, the tracking of samples to/from at the synchrotron, the visualisation of all experimental steps carried out on an individual sample and the presentation and download of the results of automatic data processing pipelines carried out at the synchrotron. This functionality is vital for remote and automated data collection but has also proven to be a powerful tool for managing experiments involving many samples and/or spanning over multiple experimental sessions. Recent improvements to EXI will be presented, including the visualization of molecular replacement structure solution for $M X$ experiments, the reporting of statistics resulting from cryo-EM image analysis and the presentation of the results of auto processing pipelines for bioSAXS experiments. 